Wagstaff, C. R. D., \& Leach, J. (2015). The value of strength-based approaches in SERE and sport psychology. Military Psychology, 27, 65-84. 


\begin{abstract}
During the past two decades, the psychosocial landscapes of the military and elite sport have drastically changed, with the presence of stress an increasing health and safety concern. For example, in modern warfare fighters must have the psychological capabilities to counter terrorism and undertake extended deployments in extreme environments against enemies with increasing skills for rapid development and dissemination of information and weaponry via global communication networks. In sport, performers have also reported being affected by globalization, reporting a hazardous array of stressors (e.g., roles, relationships, development issues, and organizational structure and climate) associated with preparation for and performance at major international competitions. The result of such developments is the need for leaders in both the military and sport to better understand optimal performance, and in particular the preparation of their charges, the war fighter and athlete, for the demands of their respective competition arenas. Therefore, in this narrative review we identify strength-based concepts of common interest and potential knowledge transfer in the study of psychology of excellence in the face of adversity between sport and military domains. Specifically, we highlight a value of six strength-based concepts for elite performance environments in sport (i.e., Olympic performance) and the military (Survival, Evasion, Resistance and Extraction: SERE); Mental toughness, positive affect, learned optimism, resilience, posttraumatic growth, and self and emotion regulation. In line with this discussion we highlight commonalities and knowledge transfer considerations regarding interventions in these spheres of psychological practice; the potential value of humanism and existentialism, the importance of post career and rehabilitation intervention, and readiness to change and engagement in training. Keywords: Strengths-based, Positive, Stress management, Performance, Survival
\end{abstract}




\section{The Value of Strength-based Approaches in SERE and Sport Psychology}

During the past two decades, the psychosocial landscapes of elite sport and the military have drastically changed. In the first of these domains, Fletcher and Wagstaff (2009) proposed that elite sport is currently embroiled in a "global sporting arms race" (p. 427) that has had far-reaching psychosocial ramifications for those operating within its sphere of influence. Part of this "arms race" relates to the power struggle between nations and major sport organizations to achieve supremacy by investing vast sums of money into performance optimization through systematic and strategic competitive advantage. In contrast, the changing landscape of the military has seen an increase in the variety of international threats as the Cold War superpowers refocus their gaze upon emerging adversaries and failed states. This has

transformed the nature of warfare and military operations and has required a diversification of skills for the warrior. To elaborate, in modern warfare fighters must have the capabilities to counter terrorism and the increasing capability for rapid development and dissemination of information and weaponry via global communication networks. Sport performers have also reported being affected by this globalization, citing a hazardous array of stressors (e.g., roles, relationships, development issues, and organizational structure and climate) associated with preparation for and performance at major competitions (see Fletcher, Hanton, Mellalieu, \& Neil, 2011). The result of such developments is the need for leaders in both sport and the military to better understand optimal performance, and in particular the preparation of their charges, the athlete and war fighter, for the demands of the competition arena. That is, psychologists operating in either domain are increasingly required to lend their expertise to facilitate the development and maintenance of high performance across a variety of tasks under conditions of extreme stress. Although one could point out the obvious issue of differences in tasks, performance conditions, and other factors (cf. Goodwin, 2008), the goal of this review is to identify areas of common interest and knowledge transfer in the study of psychology of excellence in the face of adversity from sport and military domains and discern sources of potential collaboration.

One domain that requires peak performance when confronted by adversity in the military is that of SERE psychology. SERE is a NATO acronym that stands for Survival, Evasion, Resistance and Extraction. 
It is a branch of military expertise aimed at aiding servicemen and women to survive in hostile environments and under conditions of personal extremis. Within the SERE world is the SERE psychologist who applies the skills and knowledge of psychology to the world of survival. SERE training involves placing people in conditions of environmental, physical and mental duress in order to train them to cope better physically and mentally with their circumstances and to boost their psychological health during recovery and rehabilitation. This is achieved through knowledge acquisition and skill development which combines competencies in protection, location, water and food along with stress inoculation. Basic SERE training is mostly conducted in environments such as sea, mountain, jungle, desert, polar and urban survival. It is taught to personnel who may find themselves abandoned in such regions through misadventure, such as following ship capsize or aircraft crash. Advanced level SERE training is taught to selected personnel who may find themselves not only isolated in a hostile environment but also at risk of being pursued and captured by a hostile enemy force. Specifically, such training supports those personnel who are deemed to be 'prone to capture' and involves escape and evasion techniques and methods of recovery by friendly forces; or, if captured survival within captivity and resistance to interrogation and coercion. The basic aim of SERE training is to save lives. In other words, those who have undergone SERE training should be better able to survive physical misfortune and psychological duress than most who have not had such training.

SERE training is fundamentally about human survival. Part of its stated aim in many SERE school mission statements is to foster the 'will-to-live'. In the sporting arena this can be equated to the 'will-towin' and persevere despite adverse circumstances. Part of this fostering is achieved through instilling in SERE students the appropriate knowledge (declarative memory), skills (procedural memory) and an appreciation of what to expect (episodic memory). However, the 'will-to-live' also suggests that something exists in a person above and beyond a collection of skills and competencies to aid survival. Unfortunately, the search for this 'will-to-live' has not proven fruitful and Leach (2011) has argued that the term 'will-tolive' is an outdated concept that hampers our search for a fuller understanding of human behaviour in extremis. The more interesting question is, why do so many people perish in circumstances where there is 
no need to die? The same question viewed from the perspective of sports psychology could be phrased as, why do so many sportsmen and women fail when they encounter adverse conditions when there is no need to? We might also ask why some individuals exposed to these same environmental demands appear to respond to such adversity with enhanced performance, well-being and psychological growth?

The traditional method of advanced SERE training (viz, that which includes practical resistance and conduct-after-capture training) relies heavily on stress inoculation (Meichenbaum, 1977; see for review, Saunders, Driskell, Johnston, \& Salas, 1996). This involves environmental, physical, physiological and psychological duress. It is analogous to a vaccination being given to boost a person's immunity against disease by stimulating their system without causing damage to their body or mind. Such proactive approaches are essentially designed to foster resilience amongst war fighters. Similarly, in sport, practitioners have adapted and developed stress inoculation intervention techniques and incorporated them into cognitive-behavioural intervention packages for performance enhancement (for a review, see Hanton, Thomas, \& Mellalieu, 2009). More recently, however, there has been a shift toward the examination and development of a broader range of psychosocial capacities that may facilitate optimal performance and promote well-being in adverse conditions, beyond that of stress inoculation. To illustrate the distinction between traditional and strengths-based approaches, we might return to the analogy alluded to previously; the desired outcome of the intervention is still immunity to disease - resilience - but the means to end are through the development and maintenance of a healthy organism rather than through vaccination through exposure. Indeed, whilst stress inoculation training continues to be taught to neophyte sport and performance psychology practitioners, scholars have recently called for practitioners to expand their delivery to include strengths-based approaches (see Wagstaff, Fletcher, \& Hanton, 2012a). Interestingly, the growing support for strength-based approaches to stress management in sport has also been echoed by military psychologists, with a recent special edition of the American Psychologist (Seligman \& Matthews, 2011) being dedicated to the US Army's Comprehensive Soldier Fitness (CSF) Programme. The CSF is a preventive programme that seeks to enhance psychological resilience among all members of the Army community. It provides a vision for helping those who are psychologically healthy to face life's adversities, 
including the demands of combat and prolonged separation from family using strengths-based psychological principles. Initial evaluations of the CSF program indicate that an increase in soldiers'wellness and ability to manage and bounce back from hassles, conflicts, and adversities (worthy goals) is possible for those with existing preclinical and clinical PTSD, but there is currently a lack of peerreviewed, published outcome data available on the universal prevention components of CSF (Steenkamp, Nash, \& Litz, 2013). Indeed, despite the widespread ongoing implementation of the resilience-oriented CSF, much remains to be explored regarding the potential for a range of strengths-based training for dealing with adversity in the domains of sport and the military (e.g., SERE) psychology. That is, can the development of certain psychological strengths "broaden and build” (Fredrickson, 1998) one's thoughtaction repertoires (i.e., a range of potential actions the body and mind are prepared to take) and lead to increased resources for coping with adversity? The remainder of this paper presents a narrative review of a number of purposively selected strength-based concepts which may be of interest to researchers and practitioners operating within these high performance domains.

\section{Strengths-based Psychology Concepts in SERE and Sport}

Interest in strengths-based psychology has surged with the emergence of positive psychology (Seligman \& Csikszentmihalyi, 2000). The foundations of this domain lie within human strength and flourishing research by, inter alia, Rogers (1951), Jahoda (1958), Maslow (1962), Vaillant (1977), Erikson (1982), Deci and Ryan (1985), Cooperrider and Srivastva, S. (1987), and Ryff and Singer (1996). Like these originators, positive psychologists endeavour to understand how, why, and under what conditions and processes optimal individual flourishing and functioning occurs. Hence, positive psychology is nurturing what is best in human nature and not solely fixing what is broken. In line with positive psychology, the application of such concepts has given way to the development of numerous strengths-based approaches, such as coaching. Gordon and Gucciardi (2011) define strengths-based coaching as, "the focus is on what is already working, and because strengths are part of basic human nature - everyone has strengths and deserves respect for having them - our areas of greatest potential are in the areas of our greatest strengths". The justification for such approaches is that whilst it may be possible to develop high-performance 
environments by ameliorating, correcting or eradicating weakness, improvement will only be possible once individuals, groups and organizations focus their efforts on emancipating their strengths. Beyond strengthsbased coaching, Hodges and Clifton (2004) reviewed the growing strengths-based intervention research within education, healthcare, faith communities, and business domains. Although their review includes a number of unpublished strengths-based interventions, they highlighted relationships between strengthsbased development and employee engagement, hope, subjective well-being, and confidence. More recently, Goodwin (2008) pointed to the potential collaborative efforts between sport and military psychology, stating, "of particular note are methods for understanding the development of expertise and understanding the impact of stress on performance". In line with this observation, we have selected six prominent strengths-based concepts that we feel have the potential to contribute significantly to contribute to research supporting theory and practice in both sport and military. Concepts were purposively selected that had potential to significantly influence theory and practice, an ambiguous transferability and application to sport and military domains, a strengths-based focus, and had received empirical research attention in either domain. It is our belief that the domains of SERE and sport offer excellent contexts to share, examine and apply the growing range of strengths-based concepts. Whilst obvious task differences exist between these domains, the requirement for psychological strengths to overcome adversity is very similar. In light of the potential value of these approaches, it may be beneficial to provide an overview of extant strengths-based literature from SERE and sport domains to illuminate areas of crossover across the fields of military and performance psychology.

Mental toughness. There has been considerable literature dedicated to the concept of mental toughness in elite sport performers (e.g., Gucciardi \& Gordon, 2011; Jones, Hanton, \& Connaughton, 2002). Whilst this concept has been suggested to be one of the most important psychological strengths for athletic success (Loehr, 1986), it remains one of the least understood terms in sport psychology (Jones et al., 2002). Indeed, despite 10 years of research on mental toughness, there remains some debate over its conceptualization as an outcome or a process of positive adaption to stress. However, most scholars would agree that mental toughness relates to performing to consistently high levels when under pressure and that 
it is underpinned by the ability to control stress, motivation, self-belief, and focus when under pressure (Connaughton, Thelwell, \& Hanton, 2011). If we consider this concept as an outcome, describing individuals as being "tough", we largely refer to the maintenance of competent behaviour and functionality despite the interference of emotionality under duress. Unfortunately, this does not always constitute "winning" in either sporting or SERE domains. That is, we may encounter mentally tough "losers" in sport, military operations or captivity. Conversely, if we consider mental toughness as a process, psychologists might ask why individuals are mentally tough or choose to adapt positively to stress, rather than merely how they "win" or "survive" in these extreme environments. Such a process-oriented approach to stress and performance may be of interest to SERE psychologists who believe that the pursuit of the elusive "will to survive" is misplaced and favour strengths-based developments to assist military personnel to "perform" rather than "survive".

Research aligned with the conceptualization of mental toughness as a process (see, for a review, Connaughton et al., 2011) suggests that this strength develops across three distinct stages of early, middle, and later years. During the initial developmental stage, many underlying mechanisms (e.g., a motivational climate, encouragement from significant others, and critical incidents) appear to have a powerful influence in cultivating mental toughness. Whilst it will prove difficult for SERE psychologists to optimise their students' development during this stage, we acknowledge the importance of such factors here due to their reported pertinence across the latter stages of development. Therefore, this information remains valuable for the SERE psychologist wishing to enhance mental toughness. Research by Connaughton, Wadey, Hanton, and Jones (2008) suggests that the middle years of development represented a more competitive period with which there were a range of associated demands such as, anxiety, pressure, and setbacks that may be used to an athlete's advantage if their learning can be supplemented by significant others (e.g., senior athletes, coaches, parents) to promote a heightened determination to succeed. According to Connaughton et al., in later years once mental toughness had been developed, three mechanisms are assumed to underlie the maintenance of this construct. These mechanisms relate to high levels of intrinsic and self-determined motivation, strong support networks, and the effective employment of basic and 
advanced psychological skills to help retain mental toughness. In line with the research, Gordon and Gucciardi (2011) recently highlighted the value of adopting a strengths-based coaching approach to develop and maintain mental toughness. The authors describe how a strengths-based approach was translated into practice using an example from professional cricket and conclude that such approaches may have value across age and performance levels.

In the absence of extant research examining the concept, SERE psychologists might explore the utility of incorporating the developmental nature of mental toughness into their training. That is, provide ongoing developmental opportunities which progress from the identification and evaluation of previous exposure to competitive environments (i.e., motivational climates), experiences of loss or periods of isolation (i.e., critical incidents), to the fostering of competitive environments during training, and provide assistance in the maintenance of these strengths over time. One caveat of this application of mental toughness in SERE training relates to its association with success and high performance. We believe it is important to understand that "winning" in sport does not necessarily signify a mentally tough individual and similarly so in the SERE world.

One example is that of Lance Sijan a US Air Force pilot shot down in Vietnam in 1967. Despite injuries that included a fractured leg and skull he dragged himself through the jungle for six weeks before being captured. Sijan was held with two other PoWs, Bob Craner and Guy Gruters, both recognised as 'diehards' in the PoW milieu. These two refused to answer any military questions except name, rank, service number and date of birth, until they were tortured and then they provided mostly false information. Strictly, this was going beyond the US Code of Conduct for PoWs (see DA Pam 360-512) but was recognised as a necessary escape route from debilitating torture. They counted on their resilience (known by the PoWs as 'bounce back') to enable them to recover sufficiently to reengage in the fight against their captors (see later overview of resilience). Sijan, however, stuck strictly to the letter of the Code and refused to accede anything to the enemy even under torture. His mental toughness was evident to all but, as Craner remarked he, 'was too damn brave for his own good... these people would kill him'. Sijan succumbed to the combined effects of torture, exhaustion, malnutrition and disease. Craner and Gruters survived (Howes, 
1993, p.182). This does not diminish Sijan's undoubted resistance and heroism before a brutal enemy but rather seeks to address the 'aim' of SERE training and what constitutes 'winning' or 'losing' within a SERE context.

Just as success in the sporting arena does not automatically lead to a positive, mentally healthy future for a performer, nor does surviving a hostile operation or hostage situation in the military. Rather, these outcomes are only an indication of performance. That is, a performer who encounters unrivalled success in the sporting arena may not build the necessary skills or capacities required to deal with adversity and failure that buffer against the negative effects of stress. For example, golfer Tiger Woods has often been labelled as mentally tough (e.g., Sheard, 2009), however, injury and issues from his personal life have challenged this previously unquestioned perception and required Woods to broaden his behavioural repertoire and build new skills and resources to deal with the stressors he has encountered. Similarly, a serviceman or woman who avoids capture whilst operating behind enemy lines may be considered to have "won" or inaccurately attribute this "success" to being mentally superior or tough, yet despite this performance indicator, they may not be mentally tough per se. If the individual identifies with this "tough" label it is possible that he will be more averse to seeking professional help to cope with any subsequent stress.

It is only in recent years that the military has openly acknowledged the deleterious effects of stress even on those who appear to have coped well with danger and duress. Indeed, a recent US military-funded programme of research has highlighted relationships between the concept of hardiness and physical health in military men, with this relationship being mediated by mental health (Taylor, Pietrobon, Taverniers, Leon, \& Fern, 2013) and shown positive effects of a brief psychological skills training (PST) intervention on stress responses during military survival school (Taylor et al., 2011). One cultural impediment has been the view that seeking help for psychological problems was a sign of weakness and that this could damage military careers. A recent study has found that fewer than half of military personnel returning from combat with mental health problems seek help for their disorder; the main reason cited is the public stigma associated with consulting for a mental health problem (Iverson, van 
Staden, Hughes et al., 2011). Attempts are being made to address this problem by embedding mental health personnel within military units, e.g. the OSCAR programme as established by the US Marine Corps (Nash, 2006).

Positive affect. Within strengths-based literature, Fredrickson's (1998) “broaden and build” model of positive emotion has stimulated a great deal of interest in positive emotions. This model posits that the choice to experience positive emotions broadens one's attentional focus and behavioural repertoire and, in turn, this builds and replenishes one's social, intellectual, and physical resources when dealing with stressors. A review of the broader area of affect by Pressman and Cohen (2005) highlighted consistent patterns in the literature associating positive affect and physical health with respect to morbidity, independent of negative affect. Positive emotions also appear to be important with respect to risk of mortality with several studies showing positive affect, and not negative affect, predicting survival (e.g., Moskowitz, 2003; Ostir, Markides, Black, \& Goodwin, 2000). In a series of six studies, King, Hicks, Krull, and Del Gaiso (2006) demonstrated that positive moods may predispose individuals to perceive life to be meaningful and enhance sensitivity to the relational meaning of a situation. Further, Steptoe, Gibson, Hamer, and Wardle (2007) found positive affect to be related to diurnal cortisol patterns and systolic pressure, concluding that, "positive affect is related to biological responses in the laboratory and everyday life that may be health protective" (p. 56).

In sport, the benefit of positive affect has hitherto not been wholly realized, particularly in their capacity to facilitate enhanced self-efficacy, motivation, attention, problem-solving, and coping with adversity (McCarthy, 2011). Whilst much remains to be explored regarding the importance of positive affect in sport, initial support for such explorations comes from the association of performers' moods with teammates and subjective performance in cricket. Toterdell (2000) showed significant associations between the average of teammates' happy moods and players' own moods and subjective performances; the associations were independent of hassles and favourable standing in the match. Mood linkage was greater when players were happier and engaged in collective activity. Further analyses showed that mood linkage was also greater for players who were older, more committed to the team, and more susceptible to 
emotional contagion. More recently, research by Wagstaff, Fletcher, and Hanton (2012b; 2012c) indicated that individual, group, and organizational functioning may be underpinned by psychosocial and emotionrelated skills and abilities. Moreover, the development of emotion abilities (e.g., perceiving, processing, comprehending and managing emotions) and regulation strategies (e.g., emotion experience regulation and expression regulation) has been shown to enhance organizational functioning by assisting individuals intelligently (Wagstaff, Fletcher, \& Hanton, in press). In a military setting, Maguen et al. (2008) examined risk and resilience variables among medical personnel preparing for deployment. They found predeployment positive affect to be strongly associated with resilience. The authors concluded that, “individuals exhibiting high resilience often experience positive emotions even in the throes of stressful events and compounded adversity... this creates a milieu in which military personnel are able to maintain well-being amid serious stressors" (p. 7). Thus, it is possible that positive emotion interventions could benefit SERE students and sport performers in two ways; by proactively broadening and building resilience to stressors during performance (e.g., evasion of capture or injury), and by providing guidance for reactive interventions to promote rehabilitation from adversity (e.g., recovery from capture or injury). Moreover, for service personnel or teams who are operating within SERE environments as a unit (i.e., not isolated from other personnel), the contagion effects of positive emotional experience and expression may have beneficial implications for performance and morbidity. Moreover, there appears a need to understand how to generate positive emotion in the theatre of war, where death may be encountered.

Learned optimism. A positive emotion that has been empirically associated with positive psychosocial states (e.g., Luthans, Avey, \& Patera, 2008) and mental toughness (e.g., Nicholls, Polman, Levy, Backhouse, 2008) is optimism. Similar to mental toughness, optimism has been the focus of substantial conceptual debate, with discourse centring on the nature of the concept as a dispositional trait or a state-like attribute that can be cultivated. To elaborate, Seligman (1998) referred to optimism from a positive psychological perspective as an attribution or explanatory style. Thus, Seligman, defines optimists as those who make internal, stable, and global attributions regarding positive events (e.g., survival and subsequent rehabilitation), but attribute external, unstable, and specific reasons for negative events (e.g., 
being captured or injury). In line with this conceptualisation, "learned optimism" has been contrasted with learned helplessness, which constitutes a reduced locus of control over one's situation rather than something that influences the ability to succeed. Interestingly, learned helplessness has been associated with prolonged hostage, terrorism, or political incarcerations (Busuttil, 2008), sporting success (Rettew \& Reivich, 1995) and attributions for athletic failure (Prapavessis \& Carron, 1988). Conversely, perceived stress has been found to mediate the relationship between optimism and burnout (e.g., Gustafsson \& Skoog, 2012). To the authors' knowledge, no research has specifically examined the value of learned optimism in military domains. However, elsewhere optimistic attribution styles have been associated with athletic performance (Gordon, 2008) and greater use of problem-focused and lower use of emotion-focused coping strategies during stressful events in sport (Grove \& Heard, 1997). These findings indicate that interventions to enhance optimism may be a potential way of intervening to prevent stress and burnout in athletes and soldiers as well as arming them with adaptive coping strategies when encountering stressors.

In developing approaches to assist the learning of optimistic attributions, practitioners encourage individuals to cognitively challenge negative self talk regarding imminent and impending events, and in doing so, attempt to rationalize or restructure pessimistic explanations of one's situation. It is possible that such optimism-based interventions in sport and SERE training might facilitate a more adaptive initial response to adversity allowing for enhanced affective cognitive mechanisms in working memory to assist emotion regulation (see Levens \& Gotlib, 2012) and self-regulation (Armor \& Taylor, 1998; Rasmussen, Wrosch, Scheier, \& Carver, 2006) in order to promote proactive, danger-aversive action.

A caveat of interventions to promote optimism, is that they must be coupled with the reality; for example, Viktor Frankl, whilst a prisoner in Auschwitz, observed that, 'Some men lost all hope, but it was the incorrigible optimists who were the most irritating companions' (Frankl, 2004, p. 46). However, it is no coincidence that one element of the stated ethos of the Royal Marine Commandos is 'Cheerfulness in the face of adversity'. Daily existence for a survivor comprises a series of small steps. At its very basic the day itself often has to be broken up then it has to be filled up. Peter Shaw, who was held hostage in 
Georgia, described his need to use up time and when he had used up an hour he called it a success (Shaw, 2006). These small successes increase optimism and such optimism increases survivability.

Resilience. The concept of resilience has received growing attention in sport and military domains and its literal meaning refers to the act of a substance recoiling, springing back, or resuming its original shape following bending, stretching, or compression. Most psychologists would agree that this concept refers to an individual's ability to adapt following agitation to adverse circumstances with competence by displaying relatively stable levels of psychological and physical functioning. Whilst researchers have identified resilient qualities in elite sports people and illuminated how concepts such as coping (e.g., Thelwell, Weston, \& Greenlees, 2007), social support (e.g., Rees \& Hardy, 2000), and mental toughness (e.g., Jones, Hanton, \& Connaughton, 2007) impact on performance, little research has examined the development of these qualities (see Galli \& Vealey, 2008; Richardson, 2002). More recently, Fletcher and Sarkar (2012) presented a grounded theory of resilience and optimal sport performance in a sample of Olympic champions. Results indicate that numerous psychological factors (relating to a positive personality, motivation, confidence, focus, and perceived social support) protect the world's best athletes from the potential negative effect of stressors by influencing their challenge appraisal and meta-cognitions. The authors propose that these processes promote facilitative responses that precede optimal sport performance. Non-sport literature proposes that resilience consists of three dimensions: resistance, recovery, and reconfiguration (Lepore \& Revenson, 2006). Resistance refers to being undisturbed by adversity, recovery relates to being disturbed by adversity but returning to prestress levels of functioning (e.g., returning to performance or operations), whilst reconfiguration represents being disturbed and not returning to prestress levels of functioning. To use the analogy of a tree blowing in the wind; recovery constitutes the tree being flexible and responsive to the wind, but returning to its upright position when the wind ceases; resistance reflects the tree remaining still despite strong winds; and reconfiguration represents the tree not only temporarily accommodating the wind, but adapting its shape to better deal with future storms. 
Similar to the conceptualization of resilience is that of thriving. While resilience refers to one's return to pre-stress functioning, thriving reflects a shift towards more optimal functioning as a result of the adverse experience (Wadey \& Hanton, in press). In their review on the psychology of sports injury, Wadey and Hanton (in press) propose four strategies that have been particularly useful for promoting resilience following injury: goal-setting, imagery, social support, and written emotional disclosure. They also called for greater attention to be paid to accounts of positive consequences of injury (i.e., adverse circumstances). Support for such assertions comes from Wadey, Evans, Evans, and Mitchell (2011) who examined the mechanisms that enable athletes to thrive following serious injury. Wadey et al. classified these mechanisms across three temporal phases (i.e., injury onset, rehabilitation, and return to competitive sport). The findings suggested that self-disclosure (written and/or verbal), mobilizing one's social support network, and speaking to knowledgeable others were important at injury onset. During the rehabilitation phase, the enabling mechanisms related to expanding one's social support network, strengthening one's network, increasing technical and tactical awareness and building stronger individual relationships with significant others, improving strength, conditioning, and flexibility beyond their pre-injury levels, and engaging in reflective practice on their life and priorities to gain perspective. The mechanisms during return to competitive sport included reflective practice and exposure to other distressed individuals, which resulted in athletes becoming more caring, unselfish, empathetic, and resilient. Wadey and Hanton (in press) summarise their chapter by providing suggestions for practitioners working with injured athletes, which may also provide an insight for SERE trainers. Wadey and Hanton suggest that during each phase there are several factors that should be the focus of intervention content:

Injury onset. Emotional response (i.e., encourage and reinforce the benefits of written emotional disclosure), questioning (i.e., get client to seek answers by learning about their injury), physical incapacitation (i.e., encourage clients to mobilize social support networks to assist the performance of normal daily activities).

Rehabilitation. Isolation (i.e., encourage and discuss the benefits of meeting others and engaging in reflective practice), limited autonomy and efficacy (i.e., inform clinicians and significant others of the 
importance of autonomy, supportive relationships and long- and short-term goals such as cognitive imagery to increase self-efficacy), lack of progress (i.e., offer emotional and esteem support, encourage flexibility in goals to increase motivation and recovery).

Return to competitive sport. Re-injury anxiety (i.e., encourage support network to provide positive feedback and instil confidence regarding risk situations), decision to return to sport (i.e., provide informational support and facilitate communication to ensure a safe return to sport), unrealistic expectations (i.e., challenge and replace unrealistic performance expectations using cognitive imagery to relearn previously automatic skills).

Posttraumatic growth. As alluded to above, whilst the concept of resilience has received substantial attention in psychological literature (e.g., Rutter, 1987; Bonanno, 2004), merely returning to baseline functioning following a major event for military and sport personnel is not the only potential positive outcome following traumatic experiences. Tedeschi and Calhoun (1995) coined the term posttraumatic growth to describe the positive personal changes that result from the struggle to deal with the psychological consequences associated with surviving trauma. With its foundations in humanistic and positive psychology (see Malsow, 1954; Frankl, 1963), the growing body of literature in this domain points to a number of growth areas that trauma survivors may report. To elaborate, individuals have reported renewed appreciation of life, new possibilities, enhanced personal strength, improved relationships with others, and spiritual change (Taku, Cann, Calhoun, \& Tedeschi, 2008) in response to a range of traumas including life-threatening illness and war.

In the military, empirical support for the existence of posttraumatic growth in PoWs (Erbes, et al., 2005) and combat veterans has been provided using subjective (Sledge, Boydstun, \& Rabe, 1980) and direct (Solomon \& Dekel, 2007; Maguen, Vogt, King, King, and Litz, 2006) report measures. By comparison, research on resilience in sport remains in its infancy. This is somewhat surprising given the inherent presence of adversity and stress in the sporting context (Gucciardi, Jackson, Coulter, \& Mallett, 2011). Of the extant resilience research in sport settings, scholars have examined athletes' responses to adversity regarding explanatory style (e.g., Martin-Krumm, Sarrazin, Peterson, \& Famose, 2003), 
responses to serious injury (e.g., Podlog \& Eklund, 2009) and associations between resilience, hardiness (Hanton, Neil \& Evans, in press) and burnout (Gucciardi et al., 2011). More recently, research using qualitative (Galli \& Vealey, 2008) and quantitative (Gucciardi et al., 2011) methods have lent encouraging support for the experience of positive outcomes despite exposure to adversity, by illustrating congruence, as well as domain-specific divergence with conceptualisations of resilience from other settings (cf. Werner, 1995).

In view of the encouraging findings from sport and military regarding posttraumatic growth, interventions to enhance the likelihood of growth with military personnel and sport performers should be considered. In doing so, practitioners might consider the guidelines proposed by Tedeschi and colleagues (e.g., Calhoun, Cann, \& Tedeschi, 2010; Tedeschi \& McNally, 2011). Calhoun, Cann and Tedeschi (2010) propose that the likelihood of growth may be increased by optimising several variables, including: a) cognitive processing, engagement, or rumination; b) disclosure of concerns relating to the traumatic event(s); c) the reactions of others to self-disclosures; d) the sociocultural context in which trauma occurs and attempts to process, disclose, and resolve it; e) the degree of resilience of the survivor; and f) the degree to which events permit or suppress the aforementioned processes. Further, Tedeschi and McNally (2011) propose five key elements of interventions to promote growth opportunities: a) understanding trauma response as a precursor to posttraumatic growth (i.e., understanding beliefs about self, others and future); b) emotion regulation enhancement (i.e., appraisal, control of intrusive thoughts via enhanced emotional fitness); c) constructive self-disclosure (i.e., developing a coherent trauma narrative and using social support to enhance social fitness); d) creating a trauma narrative with posttraumatic growth domains (i.e., reconfiguring shattered belief systems, disengaging from unreachable goals, and revising life narratives); and e) developing life principles that are robust to challenges (i.e., developing new ways of thinking to enhance resilience to a better level of functioning than pre-trauma).

Despite the above suggestions regarding the identification of growth opportunities, caution is required here. It must be remembered that not all survivors will experience growth following trauma. Whilst psychologists are unlikely to celebrate the experience of traumatic critical incidents (e.g., injury or 
capture) as a catalyst for growth, it is worthy of note that such incidents may set the stage for such beneficial psychological change (Tedeschi \& McNally, 2011). This sentiment has been echoed in sport psychology literature, which has highlighted the potential for critical incidents to help cultivate mental toughness (e.g., Connaughton, et al., 2008).

Self- and emotion-regulation. Like sport, SERE psychological competencies are developed through a combination of training, practice and familiarisation. Training under instruction in shelter building, fire making, radio operations, map and compass reading etc; practicing radio procedures, fire lighting, evasion methods, evacuation drills; familiarisation with environmental duress, heat, cold, wet, hunger, thirst, sleep loss etc. These are all essential elements in skill development: training is needed to acquire the skill set to aid survival; practice to develop the skill to the extent that it can be utilised without requiring higher order cognition as, in a survival situation, both attentional impairment and executive dysfunction occur (Leach \& Ansell, 2008; Porter \& Leach, 2010); familiarisation serves to remove the debilitating fear that the unknown can instil. For example, in underwater helicopter escape training the mere experience of being strapped in a seat, turned upside down in dark, swirling water means that should ditching happen for real these sensations and discomforts will no longer be novel and, therefore, will not claim substantial resources in a limited capacity cognitive system, resources that are needed for processing other vital information.

Hence, familiarisation training with an emphasis on promoting psychophysiological responses to stressors (i.e., self- or emotion-regulation) may provide an insight into the potential reasons why some individuals respond better in adverse environments than others. For example, Morgan and colleagues (Morgan et al., 2001; 2002) found SERE students who exhibited high heart-rate variability, low levels of neuropeptide Y (NPY, an amino acid involved in the regulation of noradrenergic system functioning) and baseline symptoms of dissociation performed significantly worse than comparable groups in stressinducing conditions (Doran, Hoyt, \& Moragn, 2006). Further, Morgan, Southwick, et al. (2004) found high-performing SERE students to release greater levels of dehydroepiandrosterone (DHEA, a steroid hormone that can convert oestrogen and testosterone) and NPY during stress than their poorer-performing 
counterparts. These students showed more accuracy in cognitive and memory tests after stress (Doran et al., 2006). Further, research suggests that self regulation, or the efforts by the self to alter its own responses to impulsivity during distress, is a limited mental resource with depletion leading to subsequent decreases in self regulation, vulnerability and decreased performance (see, for a recent review, Baumeister \& Alquist, 2009). Yet, there is evidence to suggest that such self-regulation and task performance can be increased with suitable training or conservation and that a "dose" of positive affect can restore the self's capacity and willingness to exert control and volition (e.g., Tice, Baumeister, Shmueli, \& Muraven, 2007).

The implications of such findings for SERE and sport psychology practitioners lie in the potential for discrete positive affect interventions to enhance self regulatory behaviours and capacities, and thus, performance, decision making, and welfare during stressful encounters.

In addition to the potential insight into psychophysiological responses those better able to selfregulate when encountering adverse situations, it is possible that emotion-related abilities might offer an insight into the propensity for individuals to approach high risk situations and how they function thereafter. To elaborate, emotional expression has been found to be associated with growth following adversity, with the relationship between event-related distress and growth mediated by emotional expression and emotional intelligence moderating the association between emotional expression and posttraumatic growth in men (Linley, Felus, Gillett, \& Joseph, 2011). Moreover, alexithymia, a largely unacknowledged personality trait, which is characterised by a difficulty in describing and identifying emotions (Taylor \& Bagby, 2000), has been shown to be strongly associated with the experience of PTSD in war veterans (see Shipko, Alvarez, \& Noviello, 1983; Hyer, Woods, Summers, Boudewyns, \& Harrison, 1990) and to moderate the anxiety experience of those engaging in high-risk sports, such as skydiving (Woodman, Huggins, Le Scanff, \& Cazenave, 2008). According to Woodman et al. (2008), given their difficulty with emotions, alexithymic individuals may find it easier to identify emotions in high-risk settings where emotions such as anxiety may be more easily identified than in other domains (e.g., social relationships). Thus, alexithymic individuals may engage in high-risk activities in order first to experience anxiety and then to experience the associated reduction in anxiety after completing the activity (Breivik et al., 1998). 
As such, high-risk operations may provide a dangerous setting for military personnel who cannot regulate their emotions. Concern exists as to whether these individuals may then be attracted to higher degrees of risk within that already dangerous environment with potentially serious implications. One particular application of this in the elite military domain relates to those seeking selection for the Special Forces, and thus undertaking SERE training. Whilst these individuals may do so by merely consenting their participation for a higher goal (i.e., Special Forces selection), it is possible that such behaviour may be underpinned by a desire for emotional stimulation for alexithymic students. This may also be applied to those who undertake military adventurous training as well as high-risk and extreme sport environments which are prone to survival and rescue interventions. For example, solo endurance sailing (e.g., Velux 5

Oceans Race), ultra marathon events (e.g., Marathon Des Sables), high altitude (e.g., climbing and extreme skiing). Hence, sport and SERE psychology training might consider the emotional regulation and alexithymia characteristics of athletes and military students.

\section{Commonalities in Intervention and Training Approaches between SERE and Sport}

In line with the preceding discussion of strength-based concepts of potential interest to psychologists working in the military and sport, we feel that there are also a number of knowledge transfer considerations regarding interventions in these spheres of psychological practice. That is, having identified six strengths suitable for knowledge transfer, we must also be cognizant of key considerations regarding the transference and application of strengths-based interventions between these domains. Hence, this section outlines three areas of mutual interest and overlap; the potential value of humanism and existentialism, the importance of post-career and rehabilitation intervention, and readiness to change and engagement in training.

The potential value of humanism and existentialism. From the SERE psychology view improvements are made by assuming life to be normal and death abnormal. A similar approach in sport psychology could be to see the experience of cognitive and somatic anxiety symptoms in response to a stressor (e.g., injury) as a common facet of the competitive environment and interpret these psychophysiological symptoms as facilitative to performance (see, for a review, Wagstaff, Neil, Mellalieu, 
\& Hanton, 2011), with negative interpretations of these symptoms being abnormal. There are exceptions in extreme survival situations such as death camps and, as Bluhm (1948) states in the opening sentence of her classic paper, 'Death in a Nazi concentration camp requires no explanation. Survival does'. To use the earlier analogy of psychological intervention or training as a vaccine, these largely existentialist approaches have proven to be a particularly difficult pill for sport and performance psychologists to swallow. Existentialists have been accused of placing too much emphasis on pessimis m, angst, and inauthenticity (Nesti, 2004). However, there is an intrinsic appeal in the empowerment that can be achieved by performers engaged in existential dialogues. For example, career transitions in sport (e.g., retirement or serious injury) will inevitably evoke questions of meaning and freedom, choice, and responsibility and transparency of self in performers. This may be another reason for the fruitless search for the "will to live" or why sport psychologists continue to rely heavily on cognitive-behavioural approaches to intervention. Indeed, neophyte practitioners are primarily taught the application of psychological skills to enhance performance and idealistic happiness-centred humanism. Existentialism might provide realism to this idealism associated with humanism. Existential sport psychologist, Mark Nesti (2004) reflected in his book:

In my work with sport performers, the search for meaning, from micro issues to the ultimate questions around life's meaning, has been the constant undercurrent. Existential Psychology is an approach for those who believe that it is only by facing up to the call to find meaning in our lives that we can find ourselves without reservation to the challenges, performances and projects we encounter daily. (xiii).

The use of the humanistic and existential paradigms to assist strengths-based practice also echoes recent calls for the use of solution-focused brief therapy (SFBT) within strengths-based coaching (see, Grant \& Cavanagh, 2011) which incorporates the use of setting concrete goals, emphasising future direction rather than past experiences, and supports clients by viewing and treating them as unique and competent, being responsive to and working with their needs.

The importance of post career and rehabilitation intervention. In comparison with non-PoW veterans, PoWs have more adjustment disorders, alcohol abuse, depressive disorders, anxiety disorders, binge eating, 
relationship difficulties, gastrointestinal and musculoskeletal disorders and premature aging (see, Doran et al., 2006). Thus, one key role of the SERE psychologist is handling repatriation of former captives and successful evaders; for example, Capt. Scott O’Grady who in June 1995 survived for almost six days evading Serb patrols after his F-16 was shot down near Mrkonjic Grad. Formal repatriation usually follows three phases. Phase 1 involves the return of the evader or captive to friendly control usually within a dedicated secure and safe area. During Phase 2 in-depth medical and psychological examination is conducted usually alongside intelligence and operational debriefs. Phase 3 involves the return of the captive to his or her own country and reuniting them with their own family as well as with groups and organisations of people who have been through similar incidents (one such British organisation is 'Hostage UK'). This phase is facilitated by the arrangement of reception and transfer processes as well as the longer processes of recuperation, reintegration, leave and return to duty. It is important to realise that a captive is not a survivor until he or she has been reintegrated into society (including, home, family, friendships, relationships and work). Recovery involves a managed return to normality and may include follow up (e.g., health monitoring and access to counselling services). Interestingly, parallels exist with sport performers recovering from serious injury or those retiring from life as an elite athlete to a normal (viz. non-elite) life. Psychological dysfunction is known to occur in both returnees and elite athletes. The former heavyweight boxer, Frank Bruno, describes this condition well; 'My trainer, George Francis, once said to me that the hardest fight would be when I retire. I didn't understand what he meant. But when you're used to getting up at seven o'clock in the morning, going running, to the gym, sparring, doing press conferences, after-parties, retiring is the worst thing that can ever happen to a sportsman' (Daily Mail, 2009). Indeed, whilst many athletes make smooth transition to retirement, researchers have expressed concern for the number of athletes who experience traumatic effects upon athletic career termination, including alcohol and substance abuse, acute depression, eating disorders, identity problems, decreased self-confidence, and attempted suicide (see Stambulova, Alfermann, Statlera, \& Côté, 2009; Wylleman, Alfermann, Lavallee, 2004). This has led to the development of athlete career education and assistance programmes to support performers in transition. The rationale for these programmes is based on the premise that by facilitating 
successful coping with transitions both within and outside of sport allows greater opportunity for an athlete to live a long and successful life in sport as well as being able to adjust effectively to the post-career (Stambulova et al., 2009). Hence, one of the foci of such programmes is the promotion and the identification of transferable skills that can be applied to other areas of an athlete's life and to other nonsport careers (Mayocchi \& Hanrahan, 2000).

One subtle difference between military and sport individuals relating to care and recovery may be in the nature of appraisal, which may reflect a sense of loss for sport performers and survival for former captives. When working with sport performers in transition, psychologists might consider using SERE analogies to promote adaptive recovery and reintegration to society. To elaborate, the counselling of "survivors" of elite sport must support developments in self-identity, locus of control, changes in emotional and social support, and the learning and enhancement of emotion regulation and coping skills. When working with athletes post-transition, more focus should be attended to voluntariness of termination, locus of control, degree of identification with the athlete role, extent of foreclosure on non-sport life areas, availability of coping resources, post-career planning, and understanding of transferable skills (Lavallee \& Anderson, 2000). Wylleman et al. (2004) reviewed the career transition programmes of national sport organizations, highlighting five common facets of these programmes: Social aspects (e.g., quality of relationship with family and friends), aspects relevant to a balanced style of living (e.g., self-image, selfesteem, self-identity, social roles and responsibilities), personal management skills (e.g., transferable and coping skills), vocational and professional occupation (e.g., vocational guidance and career advice), aspects relevant to career retirement (e.g., advantages of retirement, expected problems related to retirement, physical aspects of decreased levels of activity).

We have adapted to living in our own environment so well that we do not consider our day-to-day existence as survival. In a life-threatening or life-changing situation we are forced out of our adapted environment. There are two types of survival behaviour: intrinsic and extrinsic. Intrinsic survival is characterised by our daily, regular, routine behaviours within our normative environment. Extrinsic survival refers to those new behaviours we need to develop to survive in an environment or situation not 
previously experienced (Leach, 2011). It is also possible to become too adapted to the survival environment, a condition often referred to as 'going bush'. By becoming too adapted to the extrinsic environment it makes it difficult if not impossible to return fully to the intrinsic state and psychological dysfunction and even breakdown can be the result with clinical depression and alcoholism often the consequence. Necessarily, the extrinsic states of warfare and sport competition vary substantially, but the process of transitioning back to one's intrinsic state after an extended period of time "away" is a highly comparable obstacle experienced by military and sport personnel. Again, to quote Frank Bruno; 'I did some crazy and weird things. The pressure of everything going around just led to confusion. I felt ashamed when the ambulances came. Everybody knew what was going on except me' (Daily Mail, 2009). Such difficulty in returning to intrinsic roles and routines is also evident in standard military operational deployments of several months, which is similar to the duration sport performers may be away from their own intrinsic environment. For example, the England cricket team's 2010-2011 Ashes tour to Australia spanned five months between October 2010 and February 2011 (including a preparation period prior to departure) and Australian cricketers are reported to be spending an average of 44 weeks away from home over a three year period. This may go some large way to accounting for the increasing number of cases of severe depression being recently identified within professional cricket. Interestingly, comparatively little attention has been paid to assisting transitions amongst coaches or administrators, or those of SERE trainers.

Readiness to change and engagement in training. Here a difference often arises; whilst many elite sportsmen and women have a passion for engaging in their sport, a significant number of personnel who undergo SERE training would avoid it if at all possible. In fact, in the UK a fine distinction was drawn whereby those undergoing SERE training were no longer required to 'volunteer' for such training they merely had to 'consent' to undergo such training. For most SERE students the training they receive is never used but is considered as a behavioural accompaniment to life-saving equipment such as a life-raft or parachute. In another context, SERE training is like an insurance policy; it is hoped never to claim upon it but the premiums are still paid up front just in case. 
Whilst SERE is not a sport, can coaching lessons be learnt from sports psychology? For example, what does it mean to improve SERE training? More specifically, what does it mean to improve training for the SERE students (athletes) and their instructors (coaches)? There is an intuitive feeling that time merely spent on the job leads to improvement. To an extent it does, but mainly because most SERE students start from a low level of SERE competence and leave after a few weeks of training. This is not to say that those few weeks alone are not effective in developing coping skills sufficient for the task of survival, the evidence shows that they are. But, how can the training of SERE instructors be improved and how can we tell when it has been achieved? Those who go on in their career to qualify as SERE instructors usually have a passion for their subject that serves them well in increasing their knowledge and competencies. There exist, however, those who claim 10 years experience as a SERE instructor who in reality have only one year's experience just ten times over. To quantify such training needs an external metric and this is where sport psychology can play an important role.

How can SERE performance be measured both individually and as a team? And what is the basic team strength? The more common standard team strength is a two person unit (traditionally modelled after fast jet aircrew comprising pilot and navigator/weapon systems operator), although there will be times when SERE students will train in larger groupings as well as individuals.

Performance metrics in sport are usually well established; one knows intuitively the sort of performance standard to expect from a person classified as being novice, intermediate, proficient, expert and elite. A novice player is someone who has just taken up the sport and an elite player is someone who competes at world championship or Olympic levels. The different categories are also reasonably well defined in performance and grading for the respective sports. It could be useful to consider comparable SERE 'gradings'. This idea is suggested with the caveat that such gradings should not be overly proscriptive; in other words, the intention is to improve SERE training through understanding the methods of improvement on the sports field rather than by external regulation.

Another factor is that few SERE students want to continue with their SERE training, so even developing from novice to the next stage of 'intermediate' will be remote. It is different with the SERE 
instructors and such progression in skills, knowledge and competencies should be expected. Following this line further raises the question of the nature of SERE expertise. Is there a domain general SERE competence or are skills and abilities different across the areas of survival, evasion, resistance and extraction? Natural observation would suggest the latter. Hence, instead of considering SERE competence from the perspective of reactive strengths aligned with inoculation to stressors, survival, evasion, resistance and extraction could possibly be considered more as equivalent to a toolbox of collective and diverse psychosocial competences which incorporate strengths that have been examined in sport and outlined here. Another possible approach is suggested by 'individual psychological crisis theory (IPCT). Tenenbaum, Edmonds and Eccles (2008) state that, '[IPCT] views the athlete as a dynamic, open system that responds to environmental stimuli with certain probability levels. The athlete continuously processes information and makes decisions aimed at maximal adaptation of the system to the environmental conditions via the reduction of event uncertainty'. This quotation makes just as much sense if the word 'athlete' is replaced by the word 'survivor', thus: '[IPCT] views the survivor as a dynamic, open system that responds to environmental stimuli with certain probability levels. The survivor continuously processes information and makes decisions aimed at maximal adaptation of the system to the environmental conditions via the reduction of event uncertainty'.

IPCT enables individual probability curves of performance to be established for each sportsman and, therefore, enables improvement, or degradation, in expertise to be measured. This approach can provide a means of determining a more appropriate level of performance amongst SERE students, namely the determination of optimal performance. Given the time available for SERE training, the negligible level of previous experience and level of motivation of some SERE students, then the more practical approach would be to identify a level of optimal performance required of each SERE student and that performance to be assessed objectively through probability curves.

\section{Concluding sentiments}

This review has highlighted six areas of significant potential collaboration and knowledge transfer between military and sport psychology, it is for the psychological community to determine the contribution 
of these observations through research and practice. In returning to the US Army's Comprehensive Soldiers Fitness (CSF) programme, this proactive initiative is aimed at developing psychological resilience in soldiers, their family members and Army civilian workers. It was born out of the recognition of the deleterious effects of stress associated with sustained and protracted conflict for performance, operational readiness, and personal relationships. The programme is underpinned by strength-based psychological theory and research and is delivered at unit-level within operations and leadership to enhance cultural change. On his aspirations for CSF, US Army Chief of Staff, George W Casey stated, "my vision is that CSF becomes part of our culture over time, with our soldiers understanding the positive dimension of psychological fitness much like professional athletes do... integrated throughout our community to develop better soldiers" (Casey, 2011, p. 2). This proactive strength-based approach to psychological wellbeing and performance mimics the development of SERE and sport psychology training; however, this provision must be promoted as an organization-wide agenda as with the CSF rather than a case-by-case reactive treatment service. Indeed, it would appear that there is a great opportunity for practitioners to merge various psychological paradigms (e.g., humanistic, existential) and emerging approaches to consultancy (e.g., coaching) to better manage the challenges encountered in performance domains (e.g., career/rehab issues and readiness for behaviour change. The examination and application of strengths-based concepts in SERE and sport psychological practice may allow individuals to broaden and build their psychological awareness and encourage novel, varied, and exploratory thoughts and actions. If developed and supplemented over time, this broadened behavioral repertoire builds skills and resources that may partially buffer against the negative effects of stress arising from a poor fit between one's emotions and characteristics and the environment.

\section{References}

Armor, D. A., \& Taylor, S. E. (2003). The effects of mindset on behavior: Self-regulation in deliberative and implemental frames of mind. Personality and Social Psychology Bulletin, 29, 86-95.

Baumesiter, R., \& Alquist. J. (2009). Is there a downside to good self-control? Psychology Press, 8, 115130. 
Bluhm, H. O. (1948). How did they survive? Mechanism of defense in Nazi concentration camps. American Journal of Psychotherapy, 2, 3-32.

Bonanno, G. A. (2004). Loss, Trauma, and Human Resilience. American Psychologist, 59, 20-28.

Breivik, G., Roth, W. T., \& Jorgensen, P. E. (1998). Personality, psychological states and heart rate in novice and expert parachutists. Personality and Individual Differences, 25,365-380.

Busuttil, W. (2008). Prolonged incarceration: effects on hostages of terrorism. Journal of the Royal Army Medical Corps, 154, 128-35.

Calhoun, L. G., Cann, A., \& Tedeschi, R. G. (2010). The posttraumatic growth model: Socio-cultural considerations. In T. Weiss, R. Berger (Eds.) Posttraumatic growth and culturally competent practice: Lessons learned from around the globe. New York: Wiley.

Carver, C. S., \& Scheier, M. F. (2011). Self-regulation of action and affect. In K. D. Vohs \& R. F. Baumeister (Eds.), Handbook of self-regulation: Research, theory, and applications (2nd ed., pp. 321). New York, NY: Guilford.

Casey, G. W., Jr. (2011). Comprehensive soldier fitness: A vision for psychological resilience in the U.S. Army. American Psychologist, 66, 1-3.

Cole, P. M., Michel, M., \& Teti, L. (1994). The development of emotion regulation and dysregulation: A clinical perspective. In N. Fox (Ed.), The development of emotion regulation: Biological and behavioral considerations. Monographs of the Society for Research in Child Development, 59 (Serial no. 240).

Connaughton, D., Hanton, S., Jones, G., \& Wadey, R. (2008). Mental toughness research: Key issues in this area. International Journal of Sport Psychology, 39, 192-204.

Connaughton, D., Thelwell, R., \& Hanton, S. (2011). Mental toughness development: issues, practical implications and future directions. In D. Gucciardi \& S. Gordon (Eds.). Mental toughness in sport: Developments in research and theory (pp. 135-162). Abingdon, UK: Routledge.

Clark, L. A., Watson, D., \& Leeka, J. (1989). Diurnal variation in the positive affects. Motivation and Emotion, 13, 205-234. 
Daily Mail (2009). Depression: Top sports stars may seem to have it all - but even they are not immune. Retrieved on May 23 2012 from www.dailymail.co.uk/sport/football/article-1230687.

DA Pam 360-512. Code of the US Fighting Force. Army Pamphlet 360-512 (DoD Gen-11B). $1^{\text {st } J u n e}$ 1988.

Doran, A. P., Hoyt, G., \& Morgan, C. A. (2006). Survival, Evasion, Resistance, \& Escape (SERE) training: Preparing military members for the demands of captivity. In C. H. Kennedy \& E. A. Zillmer (Eds.), Military psychology: Clinical and operational applications (pp. 241-261). New York: Guilford Press.

Erbes, C., Eberly, R., Dikel, T., Johnsen, E., Harris, I., \& Engdahl, B. (2005). Posttraumatic growth among American former prisoners of war. Traumatology, 11, 285-295.

Fletcher, D., Hanton, S., Mellalieu, S. D., \& Neil, R. (2011). A conceptual framework for organizational stressors in sport performers. Scandinavian Journal of Medicine and Science in Sports.

Fletcher, D., \& Sarkar, M. (2012). A grounded theory of psychological resilience in Olympic champions. Psychology of Sport and Exercise, 13, 669-678.

Fletcher, D., \& Wagstaff, C. R. D. (2009). Organizational psychology in elite sport: Its emergence, application and future. Psychology of Sport and Exercise, 10, 427-434.

Frankl, V. E. (1985). Man's search for meaning. New York Washington Square Press.

Frankl, V. E. (2004). On the Theory and Therapy of Mental Disorders. New York: Routledge.

Fredrickson, B. L. (1998). What good are positive emotions? Review of General Psychology, 2, 300-319.

Galli, N., \& Vealey, R. S. (2008). Bouncing back from adversity: Athletes' experiences of resilience. The Sport Psychologist, 22, 316-335.

Gordon, R. A. (2008). Attributional style and athletic performance: Strategic optimism and defensive pessimism. Psychology of Sport and Exercise, 9, 336-350.

Gordon, S., \& Gucciardi, D. F. (2011). Strengths-based approach to coaching mental toughness. Journal of Sport Psychology in Action, 2, 143-155 
Grant, A. M., \& Cavanagh, M. (2011). Coaching and positive psychology. Designing positive psychology: Taking stock and moving forward, 293-309.

Grove, J. R., \& Heard, N. P. (1997). Optimism and sport confidence as correlates of slump-related coping among athletes. The Sport Psychologist, 11, 400-410.

Gucciardi, D. F., \& Gordon, S. (2011). Mental toughness in sport: Developments in research and theory. Abingdon, UK: Routledge.

Gucciardi, D. F., Jackson, B., Coulter, T. J., \& Mallett, C. J. (2011). The Connor-Davidson Resilience Scale (CD-RISC): Dimensionality and age-related measurement invariance with Australian cricketers. Psychology of Sport and Exercise, 12, 423-433.

Hanton, S., Neil, R., \& Evans, L. (in press). Hardiness and anxiety interpretation: An investigation into coping usage and effectiveness. European Journal of Sports Sciences.

Hanton, S., Thomas, O., \& Mellalieu, S. D. (2009). Management of competitive stress in elite sport. In B.W. Brewer (Ed.). International Olympic Committee sport psychology handbook (pp. 30-42). Oxford, UK: Blackwell Publishing.

Hodges, T. D., \& Clifton, D. O. (2004). Strengths-based development in practice. In P. A. Linley \& S. Joseph (Eds.), International handbook of positive psychology in practice: From research to application (pp. 256-268). New York: Wiley.

Howes, C. (1993). Tales of the Vietnam PoWs: Witnesses to their fight. New York: Oxford University Press.

Hyer, L., Woods, G. M., Summers, M. N., Boudewyns, P., Harrison, W. R. (1990). Alexithymia among Vietnam veterans with posttraumatic stress disorder. Journal of Clinical Psychiatry, 51, 243-247.

Iverson, A., van Staden, L., Hughes, J., Browne, T., Hull, I., Hall, J., et al. (2009). The prevalence of common mental disorders and PTSD in the UK military: using data from a clinical interview-based study. BMC Psychiatry, 9, 68.

Jahoda, M. (1958). Current concepts of positive mental health. New York: Basic Books. 
Jones, G., Hanton, S., \& Connaughton, D. (2002). What is this thing called Mental Toughness?: An investigation with elite performers. Journal of Applied Sport Psychology, 14, 211-224.

Jones, G., Hanton, S., \& Connaughton, D. (2007). Mental Toughness: An examination of Olympic and world champion performers. The Sport Psychologist, 21, 243-264.

King, L. A., Hicks, J. A., Krull, J., \& Del Gaiso, A. K. (2006). Positive affect and the experience of meaning in life. Journal of Personality and Social Psychology, 90, 179-196.

Lavallee, D., \& Andersen, M. B. (2000). Leaving sport: easing career transitions. In M. B. Andersen (Ed.), Doing sport psychology (pp. 249-260). Champaign, IL: Human Kinetics.

Leach, J. (2011). Survival psychology: The won’t to live. The Psychologist, 24, 26-29.

Leach, J., \& Ansell, L. (2008). Impairment in attentional processing in a field survival environment. Applied Cognitive Psychology, 22, 643-652.

Lepore, S. J., \& Revenson, T. A. (2006). Resilience and posttraumatic growth: Recovery, resistance, \& reconfiguration. In L. Calhoun R. G. Tedeschi (Eds.), The Handbook of Posttraumatic Growth: Research and Practice (pp. 24-46). Mahwah, NJ: Erlbaum.

Levens, S. M., Gotlib, I. H. (2012). The effects of optimism and pessimism on updating emotional information in working memory. Cognition and Emotion, 26, 341-350.

Linley, P. A., Felus, A., Gillett, R., \& Joseph, S. (2011). Emotional expression and growth following adversity: Emotional expression mediates subjective distress and is moderated by emotional intelligence. Journal of Loss and Trauma: International Perspectives on Stress \& Coping, 16, 387401.

Loehr, J. E. (1986). Mental toughness training for sports: achieving athletic excellence. Lexington, MA: Stephen Greene Press.

Luthans, F., Avey, J. B., \& Patera, J. L. (2008). Experimental analysis of a web-based training intervention to develop positive psychological capital. Academy of Management Learning and Education, 7, 209-221. 
Maguen, S., Vogt, D. S., King, L. A., King, D. W., \& Litz, B. T. (2006). Posttraumatic growth among Gulf War I veterans: The predictive role of predeployment, deployment and postdeployment factors. Journal of Loss and Trauma, 11, 373-388.

Martin-Krumm, C., Sarrazin, P., Peterson, C., \& Famose, J. P. (2003). Explanatory style and resilience after sports failure. Personality and Individual Differences, 35, 1685-1695.

Maslow, A. H. (1962). Toward a psychology of being. Princeton, NJ: Van Nostrand.

Mayocchi, L., \& Hanrahan, S. J. (1997). Adaptation to a post-athletic career: The role of transferable skills. Belconnen, ACT: Australian Sports Commission.

McCarthy, P. J. (2011). Positive emotion in sport performance: current status and future directions. International Review of Sport and Exercise Psychology, 4, 50-69.

Meichenbaum, D. (1977). Cognitive behaviour modification: An integrative approach. Plenum Press, New York.

Morgan, C. A., Southwick, S., Hazlett, G., Rasmusson, A., Hoyt, G., Zimolo, Z., et al. (2004). Relationships among plasma dehydroepiandrosterone in humans exposed to acute stress. Archives of General Psychiatry, 61, 819-825.

Morgan, C. A., Wang, S., Rasmusson, A., Hazlett, G., Anderson, G., \& Charney, D. (2001). Relationship among plasma cortisol, catecholamines, neuropeptide-Y, and human performance during exposure to uncontrollable stress. Psychosomatic Medicine, 63, 412-422.

Morgan, C. A., Rasmusson, A., Wang, S., Hoyt, G., Hauger, R., \& Hazlett, G. (2002). Neuropeptide-Y, cortisol, and subjective distress in humans exposed to acute stress: Replication and extension of previous report. Biological Psychiatry, 52, 136-142.

Moskowitz, J. T. (2003). Positive affect predicts lower risk of AIDS mortality. Psychosomatic Medicine, 65, 620-626.

Nash, W. P. (2006). Operational Stress Control and Readiness (OSCAR): The U.S. Marine Corps Initiative to Deliver Mental Health Services to Operating Forces. Human Dimensions in Military Operations- 
Military Lead-ers' Strategies for Addressing Stress and Psychological Support. Nesti, M. (2004). Existential psychology and sport. London, UK: Routledge.

Nicholls, A. R., Polman, R. C. J., Levy, A. R., Backhouse, S. H. (2008). Mental toughness, optimism, pessimism, and coping among athletes. Personality and Individual Differences, 44, 1182-1192.

Ostir, G. V., Markides, K. S., Black, S. A., \& Goodwin, J. S. (2000). Emotional well-being predicts subsequent functional independence and survival. Journal of the American Geriatrics Society, 48, 473-478.

Podlog, L., \& Eklund, R. C. (2009). High-level athletes' perceptions of success in returning to sport following injury. Psychology of Sport and Exercise, 10, 535-544.

Porter, H., \& Leach, J. (2010). Executive dysfunction in a survival environment. Applied Cognitive Psychology, 24, 41-66.

Prapavessis, H., \& Carron, A. V. (1988). Learned helplessness in sport. The Sport Psychologist, 2, 189201.

Pressman, S. D., \& Cohen, S. (2005). Does positive affect influence health? Psychological Bulletin, 131, 925-971.

Rasmussen, H. N., Wrosch, C., Scheier, M. F., \& Carver, C. S. (2006). Self-regulation processes and health: The importance of optimism and goal adjustment. Journal of Personality, 74, 1721-1747.

Rees, T., \& Hardy, L. (2000). An investigation of the social support experiences of high-level sports performers. The Sport Psychologist, 14, 327-347.

Reivich, K. (1995). The measurement of explanatory style. In G. M. Buchanan, \& M. E. P. Seligman (Eds.), Explanatory style (pp. 21-47). Hillsdale, NJ: Erlbaum.

Richardson, G. E. (2002). The metatheory of resilience and resiliency. Journal of Clinical Psychology, 58, 307-321.

Rogers, C. R. (1951). Client-centered therapy: Its current practice, implications, and theory. Boston: Houghton Mifflin. 
Rutter, M. (1987). Psychosocial resilience and protective mechanisms. American Journal of Orthopsychiatry, 57, 316-331.

Ryff, C. D., \& Singer, B. (1996). Psychological well-being: Meaning, measurement, and implications for psychotherapy research. Psychotherapy and Psychosomatics, 65, 14-23.

Saunders, T., Driskell, J. E., Johnston, J. H., \& Salas, E. (1996). The effect of stress inoculation training on anxiety and performance. Journal of occupational health psychology, 1, 170.

Seligman, M. E. P. (1998). President's column: Positive social science. APA Monitor, 29, retrieved on December 13, 2011 from www.apa.org/monitor/apr98/pres.html

Seligman, M. E. P., \& Csikszentmihalyi, M. (2000). Positive psychology: An introduction. American Psychologist, 55, 5-14.

Seligman, M. E. P., \& Matthews, M. D. (Eds.) (2011). Comprehensive Soldier Fitness: Special issue of the journal. American Psychologist, 66.

Shaw, P. (2006). Hole: Kidnapped in Georgia. Pembrokeshire, UK: Accent Press Ltd.

Sheard, M. (2009). Mental toughness: The mindset behind sporting achievement. London, UK: Routledge.

Shipko, S., Alvarez, W. A., \& Noviello, N. (1983). Towards a teleological model of alexithymia: alexithymia and post-traumatic stress disorder. Psychotherapy and psychosomatics, 39, 122-126.

Sledge, W., Boydstun, J., \& Rabe, M. (1980). Self-concept changes related to war captivity. Archives of General Psychiatry, 37, 430-443.

Solomon, Z., \& Dekel, R. (2007). Posttraumatic stress disorder and posttraumatic growth among Israeli exPoWs. Journal of Traumatic Stress, 20, 303-312.

Stambulova, N., Alfermann, D., Statler, T., \& Côté, J. (2009). Career development and transitions of athletes: the ISSP position stand. International Journal of Sport \& Exercise Psychology, 7, 395412.

Steenkamp, M. M., Nash, W. P., \& Litz, B. T. (2013). Post-traumatic stress disorder: Review of the comprehensive soldier fitness program. American Journal of Preventive Medicine, 44, 507-512. 
Steptoe, A., Gibson E. L., Hamer M., \& Wardle, J. (2007). Neuroendocrine and cardiovascular correlates of positive affect measured by ecological momentary assessment and by questionnaire. Psychoneuroendocrinology, 32, 56-64.

Taku, K., Cann, A., Calhoun, L. G., \& Tedeschi, R. G. (2008). The factor structure of the posttraumatic growth inventory: A comparison of five models using confirmatory factor analysis. Journal of Traumatic Stress, 21, 158-164.

Taylor, G., \& Bagby, R. (2000). An overview of the alexithymia construct. In R. Bar-On and J. Parker (Eds.), The handbook of emotional intelligence (pp. 40-67). San Francisco, CA: Jossey-Bass.

Taylor, M. K., Pietrobon, R., Taverniers, J., Leon, M. R., \& Fern, B. J. (2011). Relationships of hardiness to physical and mental health status in military men: a test of mediated effects. Journal of Behavioral Medicine, 1-9.

Taylor, M. K., Stanfill, K. E., Padilla, G. A., Markham, A. E., Ward, M. D., Koehler, M. M., ... \& Adams, B. D. (2011). Effect of psychological skills training during military survival school: A randomized, controlled field study. Military Medicine, 176, 1362-1368.

Tedeschi, R. G., \& Calhoun, L. G. (1995). Trauma and transformation. California: Sage.

Tedeschi, R. G., \& McNally, R. J. (2011). Can we facilitate posttraumatic growth in combat veterans? American Psychologist, 66, 19-24.

Tenenbaum, G., Edmonds, W. A., \& Eccles, D. W. (2008). Emotions, coping strategies, and performance: A conceptual framework for defining affect-related performance zones. Military Psychology, 20, 11-37.

Thelwell, R. C., Weston, N. J. V., \& Greenlees, I. A. (2007). Batting on a sticky wicket: Identifying sources of stress and associated coping responses for professional cricket batsmen. Psychology of Sport and Exercise, 8, 219-232.

Tice, D. M., Baumeister, R. F., Shmueli, D., \& Muraven, M. (2007). Restoring the self: Positive affect helps improve self-regulation following ego depletion. Journal of Experimental Social Psychology, $43,379-384$. 
Totterdell, P. (2000). Catching moods and hitting runs: Mood linkage and subjective performance in professional sport teams. Journal of Applied Psychology, 85, 848-859.

Vaillant, G. E. (1977). Adaptation to life. Boston: Little, Brown.

Wadey, R., Evans, L., Evans, K., \& Mitchell, I. (2011). Perceived benefits following sport injury: A qualitative examination of their antecedents and underlying mechanisms. Journal of Applied Sport Psychology, 23, 142-158.

Wadey, R., \& Hanton, S. (in press). Psychology of sport injury: Resilience and thriving. In F. G. O’Conner and R. Wilder (Eds.), Running Medicine. New York: McGraw Hill.

Wagstaff, C. R. D., Fletcher, D., \& Hanton, S. (2012a). Positive organizational psychology in sport: An ethnography of organizational functioning in a national sport organization. Journal of Applied Sport Psychology, 24, 26-47.

Wagstaff, C. R. D., Fletcher, D., \& Hanton, S. (2012b). Positive organizational psychology in sport. International Review of Sport and Exercise Psychology. ISSN 1750-984X 10.1080/1750984X.2011.634920

Wagstaff, C. R. D., Fletcher, D., \& Hanton, S. (2012c). Exploring emotion abilities and regulation strategies in sport organizations. Sport, Exercise and Performance Psychology.

Wagstaff, C. R. D., Hanton, S., \& Fletcher, D. (in press). Developing emotion abilities and regulation strategies in a sport organization: an action research intervention. Psychology of Sport \& Exercise.

Wagstaff, C. R. D., Neil, R., Mellalieu, S. D., \& Hanton, S. (2011). Key movements in directional research in competitive anxiety. In J. Thatcher, M. M. Jones and D. Lavallee (Eds.). Coping and emotion in sport (pp.143-166). Routledge.

Werner, E. E. (1995). Resilience in development. Current Directions in Psychological Science, 4, 81-85. 
Table 1. A Summary of strength-based concepts for potential knowledge transfer between sport and military domains

Strength-based concept Definition

Mental toughness

Mental toughness refers to performing to consistently high levels

when under pressure and is underpinned by the ability to control stress, motivation, self-belief, and focus when under pressure (adapted from Connaughton, Thelwell, \& Hanton, 2011)

Positive affect Positive affect reflects a composite of mood, emotion and feelings emotion or affect as feelings that are valenced by a level of pleasurable engagement with the environment. Those experiencing high positive affect feels enthusiastic, active, and alert (adapted from Clark, Watson, \& Leeka, 1989)

Learned optimism Learned optimism refers to the notion that individuals can learn to use adaptive causal attributions to explain negative experiences and events. It is characterized by making external, variable, and specific attributions for failure or traumatic events rather than the internal, stable, and global attributions of the pessimist. It is contrasted with learned helplessness.

Resilience Resilience is an individual's ability to adapt following agitation to adverse circumstances with competence by displaying relatively stable levels of psychological and physical functioning. It is characterized by three dimensions: resistance (e.g., being undisturbed by adversity), recovery (e.g., returning to performance or operations), and reconfiguration (e.g., not returning to pre-stress 
levels of functioning) (adapted from Lepore \& Revenson, 2006).

Posttraumatic growth

Self and emotion regulation
Posttraumatic growth reflects the positive personal changes that result from the struggle to deal with the psychological consequences associated with surviving trauma. This is distinguished from resilience by reflecting positive outcomes above and beyond those that characterized pre-trauma functioning.

Self regulation conveys a range of purposive processes which provide a sense that self-corrective adjustments are taking place as needed to stay on track regarding goals, norms and rules (whether this entails overriding another impulse or simply reacting to perturbations from other sources), and the sense that the corrective adjustments originate within the person (adapted from Carver \& Scheier, 2011). Emotion regulation might be defined as the ability to respond to the ongoing demands of experience with the range of emotions in a manner that is socially tolerable and sufficiently flexible to permit spontaneous reactions as well as the ability to delay spontaneous reactions as needed (adapted from Cole, Michel \& Teti, 1994). That is, it represents the ways in which emotions are regulated (e.g., cognitive control, internalization of social expectations) to allow individuals to monitor, delay, and adjust preparatory reactions and adapt them to the complexities and subtleties of situational demands. 